\title{
Three-Dimensional Discrete Element Simulations of Direct Shear Tests
}

\author{
Catherine O'Sullivan, Liang Cui \\ Department of Civil Engineering, University College Dublin, Ireland \\ Jonathan D. Bray \\ Department of Civil and Environmental Engineering, University of California, Berkeley, USA
}

\begin{abstract}
Using discrete element simulations, one can monitor the micro-mechanisms driving the macroresponse of granular materials and quantify the evolution of local stress and strain values. However, it is important to couple the se simulations with carefully controlled physical tests for validation and insight. Only then can findings about the micro-mechanics of the material response be made with confidence. Moreover, the sensitivity of the observed response to the test boundary conditions can be analyzed in some detail. The results of three-dimensional discrete element simulations of direct shear tests and as well as complementary physical tests on specimens of steel balls are presented in this paper. Previous discrete element analyses of the direct shear test have been restricted to two-dimensional simulations. For the simulations presented here, an analysis of the internal stresses and contact forces illustrates the three-dimensional nature of the material response. The distribution of contact forces in the specimen at larger strain values, however, was found to be qualitatively similar to the two-dimensional results of Zhang and Thornton (2002). Similarities were also observed between the distrib ution of local strain values and the distribution of strains obtained by Potts et al (1987) in a finite element analysis of the direct shear test. The simulation results indicated that the material response is the stress dependent. However, the response observed in the simulations was found to be significantly stiffer than that observed in the physical tests. The angle of internal friction for the simulations was also about $3^{\circ}$ lower than that measured in the laboratory tests. Further laboratory tests and simulations are required to establish the source of the observed discrepancies.
\end{abstract}

\section{INTRODUCTION}

To date, much of our understanding of soil $\mathbf{e}$ sponse has developed using standard laboratory tests. Much can be learned by revisiting these tests and examining the micro-mechanics underlying the observed macro-scale response using discrete element method (DEM) analyses of these tests. This paper presents some preliminary results of an ongoing analysis of the direct shear test that couples physical tests and DEM simulations. The physical tests on stainless steel spheres are described firstly. Following a detailed description of the details of the DEM simulation approach, the numerical results are compared with the physical tests results. Significant differences in the material responses were observed. The next phase of this research will attempt to identify the source of these differences. Analysis of the simulation results examined the local stresses, the particle displacements, the contact forces and the bcal strains. These results are compared with the earlier findings of Zhang and Thornton (2002) and Potts et al (1987).

\section{BACKGROUND}

Although there appears to be a slight difference in opinion on the origin of the direct shear test, it is unquestionably one of the oldest and most commonly used laboratory tests in geotechnical engineering. Holtz and Kovacs (1981) state that the tests was originally used by Coulomb in the $18^{\text {th }}$ century, while Potts et al (1987) cite Skempton (1949) who found that the test was first used in the $19^{\text {th }}$ century by Alexandre Collin. In a preface to a Geotechnique symposium in print on "The engineering application of direct and simple shear testing," Toolan (1987) observed that the direct shear test declined in popularity with the emergence of triaxial testing. Toolan argues that testing with a direct shear test provides essential data for applications with low factors of safety where moderate displacements of the soil can be expected upon failure. A recent publication by Lings and Dietz (2004) proposes modifications to the apparatus to facilitate testing of sand, illustrating 
continued interest in this apparatus. Other more recent research includes the experimental studies by Shibuya et al (1997) and the finite element analyses described by Dounias et al (1993) and Potts et al (1987).

The standard test procedure for the direct shear test is documented in ASTM D3080 (1990). Both Potts et al (1987) and Shibuya et al (1997) note the limitations of the test, including the non-uniformity of the stresses and strains induced in the soil and difficulties associated with interpreting the test results to define a failure criterion. As noted by both Potts et al and Shibuya et al, the popularity of the test can largely be attributed to its simplicity.

Shibuya et al (1997) examined the influence of boundary effects, such as wall friction, on the specimen response. The distribution of normal and shear stresses in the specimens tested were measured using two-component load cells. Considering direct shear tests on specimens of dense Toyoura sand the effect of wall friction on the specimen response were studied. It was found that as a result of wall friction, vertical stresses on the potential shear plane were found to be substantially lower than the conventional measurement of vertical stress and that the value of $\phi$ at peak was over-estimated when the stress was calculated from the applied normal force directly.

Potts et al (1987) and Dounias et al (1993) performed continuum-based numerical analyses of the test using the finite element method. Potts et al found that the reference stress state for the direct shear box is that of simple shear. The study also considered the influence of $\mathrm{K}_{0}$ on the specimen response. When a constitutive model without strain softening was used to describe the material response, the distribution of stresses and strains within the material were highly non-uniform. However when a model incorporating significant strain softening was used, the stresses and strains were more uniform.

Some earlier research has explored the applicability of DEM to simulate the direct shear test. Morgan and Boettcher (1999) and Morgan (1999) used the DEM code TRUBAL to simulate direct shear tests with the objective of understanding the nature of deformation of granular fault gouge. Morgan and Boettcher calculated the directional derivative of the horizontal displacement surface as a means to highlight discontinuities in the specimen. They observed that at lower strain values (about 2\%) the deformation was broadly distributed across multiple subhorizontal slip planes and progressively localized onto a single shear plane by $8-10 \%$ strain.
Masson and Martinez (2001) used the twodimensional code PFC2D (Itasca, 2002) to simulate two-dimensional direct shear tests using 1050 disks of diameter 2, 3 and $4 \mathrm{~mm}$. The particle scale responses analysed by Masson and Martinez included the particle instantaneous velocity field, the distribution of particle horizontal and vertical displacements as a function of depth and the distribution of particle rotation as a function of depth. As other studies have shown (e.g. Iwashita and Oda, 1998) the particle rotations were found to be a significant indicator of strain localization. Masson and Martinez recognized the limitations of their two-dimensional study and highlighted the potential sensitivity of their results to the particle-boundary friction value.

In another two-dimensional study, using a single layer thickness of 5000 polydisperse spherical particles, Zhang and Thornton (2002) also analysed the direct shear test using the TRUBAL code. Comparing the findings of Zhang and Thornton and Masson and Martinez, the orientation of the contact forces at the peak strength exhibit a more definite trend in the simulation of Zhang and Thornton, most likely as a consequence of the larger number of particles used.

All of these earlier studies of the direct shear test using DEM considered two-dimensional analyses. The work of Thomas (1997) quantitatively demonstrates the limitations of both two-dimensional DEM simulations and physical tests using "Schneebli" rods to represent real soil response. The current study builds upon the findings of these earlier researchers by extending the simulations to three dimensions. Furthermore, by coupling the numerical simulations with complementary physical tests, further insight into the material response can be obtained.

\section{PHYSICAL TESTS}

\subsection{Direct shear testing}

A schematic diagram of the direct shear test apparatus used in the current study is given in Figure 1. The apparatus comprises a metal box of square cross section (60 $\mathrm{mm}$ wide), divided in two halves horizontally. The lower section of the box was moved forward at a constant velocity $(0.015 \mathrm{~mm} / \mathrm{s})$ while the upper section of the box remained stationary. The force required to maintain the upper section of the box in a stationary position was measured using a load cell and proving ring. The calibration of the load cell was checked prior to testing. The 
vertical load was applied to the top of the shear box using a system of dead weights attached to a lever.

In the current study $0.9922 \mathrm{~mm}$ Grade 25 Chrome Steel Balls were used (manufacturer: Thompson Precision Ball). This material was used as the to erances on the ball diameter and sphericity were relatively tight $\left( \pm 7.5 \times 10^{-4} \mathrm{~mm}\right)$. The ball material properties are summarized in Table 1 . The coefficient of friction values obtained by O'Sullivan (2002) are used in the current study as the steel balls used are equivalent to the steel balls considered in the earlier study. For each test the balls were placed in the shear box in three equal layers. Each layer had a mass of $125 \mathrm{~g}$ (corresponding to 3900 balls). Following placement of the balls, five "taps" of a hammer were applied to each vertical side of the box to increase the specimen density.

The test results are illustrated in Figure 2 and summarized in Table 2. The shear stress was calculated by dividing the horizontal load measured in the proving ring by the specimen cross sectional area, while the vertical stress was calculated by dividing the applied vertical load by the specimen cross-sectional area. As illustrated in Figure 2(b), the friction angle $\phi$ for the material was found to be $24.4^{\circ}$. However, as illustrated in both Figure 2(a) and Figure 2(b), there was a degree of scatter in the experimental results. By reference to Table 2 it can be seen that the variation in specimen response appears to be largely a result of variation in the specimen void ratio values which were difficult to control accurately. Considering the maximum shear stresses and the minimum shear stresses measured for each normal stress, the maximum friction angle was found to be $26.2^{\circ}$, while the minimum friction angle was found to be $22.7^{\circ}$. To verify the experimental results the tests were repeated in the geotechnical laboratory at Trinity College Dublin. For specimens with equivalent densities, the measured friction angles differed by less than $0.25^{\circ}$.

\begin{tabular}{|l|l|l|}
\hline Parameter & Value & Unit \\
\hline Radius & 0.9922 & $\mathrm{~mm}$ \\
\hline Density & $7.8334 \times 10^{-6}$ & $\mathrm{~kg} / \mathrm{mm}^{3}$ \\
\hline Shear modulus & $7.945 \times 10^{\prime}$ & $\mathrm{kg} /\left(\mathrm{mm} \mathrm{s}^{2}\right)$ \\
\hline Poisson ratio & 0.28 & \\
\hline Friction coefficient* & $5.5^{\circ}$ & \\
\hline
\end{tabular}

Table 1: Material parameters of Grade 25 chrome steel balls used in laboratory tests. *As determined by O'Sullivan (2002) for equivalent balls

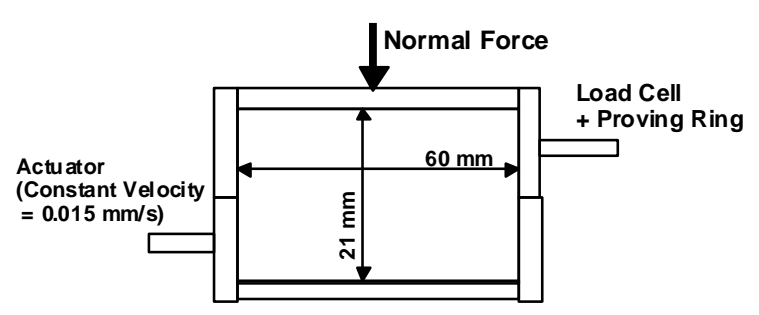

Figure 1: Schematic diagram of direct shear test set-up

\begin{tabular}{|l|ll|l|}
\hline Test No. & $\begin{array}{l}\text { Normal } \\
\text { Stress } \\
(\mathrm{kPa})\end{array}$ & $\begin{array}{l}\text { Peak Shear } \\
\text { Stress }(\mathrm{kPa})\end{array}$ & Void Ratio \\
Dense 1-1 & 54.5 & 24.86 & 0.5841 \\
\hline Dense 1-2 & 54.5 & 27.23 & 0.5826 \\
\hline Dense 1-3 & 54.5 & 19.58 & 0.6089 \\
\hline Dense 2-1 & 109 & 45.90 & 0.5991 \\
\hline Dense 2-2 & 109 & 49.50 & 0.6097 \\
\hline Dense 2-3 & 109 & 42.75 & 0.5916 \\
\hline Dense 3-1 & 163.5 & 83.03 & 0.5818 \\
\hline Dense 3-2 & 163.5 & 71.55 & 0.6044 \\
\hline Dense 3-3 & 163.5 & 75.15 & 0.5969 \\
\hline
\end{tabular}

Table 2: Summary of physical test results

\section{DEM SIMULATIONS}

\subsection{Implementation of direct shear testing in 3DDEM}

The distinct element method program (3DDEM) used for the simulations described here is a modification of a code developed by $\mathrm{Lin}$ and $\mathrm{Ng}$ (1997) for three-dimensional ellipsoidal particles, which in turn is a modification of the Trubal DEM code (Cundall and Strack, 1979). The program uses spherical particles and is further described in O'Sullivan (2002). In the DEM code, the Hertz-Mindlin contact model is used to model the contact between spheres. Details of the implementation of this model can be found in Itasca (2002) or Bardet (1998).

The method of simulating the direct shear test in the 3DDEM code was originally proposed by O'Sullivan (2002). A diagram illustrating the simulation procedure is given in Figure 3. During the simulation, the specimen is enclosed by eight rigid boundaries, two horizontal boundaries at the top and bottom, two vertical boundaries at the front and back, and four vertical boundaries at the left and right of the specimen. Prior to shearing, the two left boundaries are co-planar and the two right boundaries are co-planar. During shearing, one set of vertical boundaries is moved laterally with a constant velocity. An imaginary horizontal plane is constructed through the middle of the specimen. All of the particles with centroids located above this plane are checked for contact with the moving boundaries, 
while all of the particles with centroids located below this plane are checked for contact with the stationary boundaries. Using this approach the computational cost associated with contact detection between a sphere and the edge of the shear box is avoided.

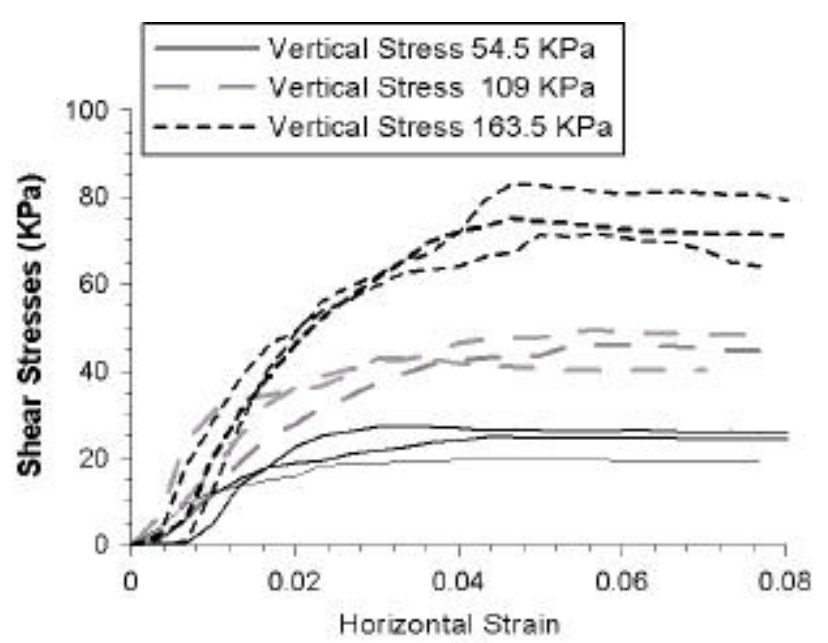

(a)

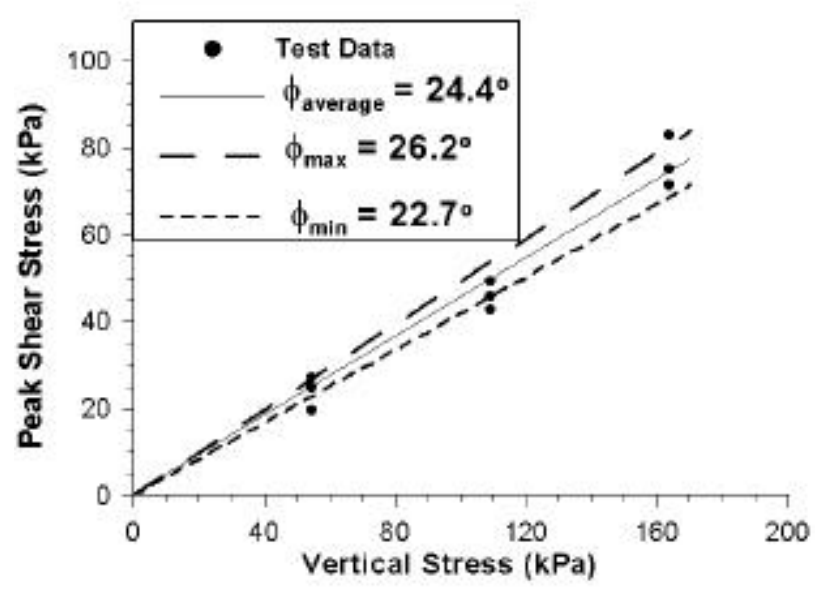

(a)

Figure 2: Summary of physical test results, (a) Shear stress versus horizontal strain (b) Shear stress versus normal stress

The measurement sphere illustrated in Figure 3 is used to measure the average stresses in the specimen. Such a system to measure stress in discrete element simulations has been described by a number of researchers including Bardet (1998). The average stress $\bar{\sigma}_{i j}$ in the sphere is given by

$$
\bar{\sigma}_{i j}=\frac{1}{V} \sum_{c=1}^{N c} f_{i}^{c} l_{i}^{c} \quad(\mathrm{i}, \mathrm{j}=\mathrm{x}, \mathrm{y}, \mathrm{z})
$$

where $N_{c}$ is the number of contacts within the measurement sphere, $V$ is the volume of the sphere, $f_{i}^{c}$ is the contact force at contact $c, l_{i}^{c}$ is the branch vector connecting two contacted particle, a and $b$. The branch vector is given by

$$
l_{i}^{c}=x_{i}^{b}-x_{i}^{a} \quad \mathrm{i}, \mathrm{j}=\mathrm{x}, \mathrm{y}, \mathrm{z}
$$

where $x^{a}$ and $x^{b}$ are the position vectors of the spheres $\mathrm{a}$ and $\mathrm{b}$ meeting at contact $\mathrm{c}$.

Prior to shearing the measurement sphere is used in a servo-controlled system to bring the specimen into a prescribed initial stress configuration. If the stress measured within the measurement sphere, $\sigma_{i i}^{\text {meas }}$, differs from the user-specified stress, $\sigma_{i i}^{\text {req }}$, the boundaries perpendicular to $i$ direction are slowly moved so that the measured stress attains the required stress values. A servo-controlled approach is also used during the test so that the vertical stress, as measured in the measurement sphere, remains constant during shearing. A detailed description of the implementation of the servocontrolled system in 3DDEM is given by O'Sullivan (2002).

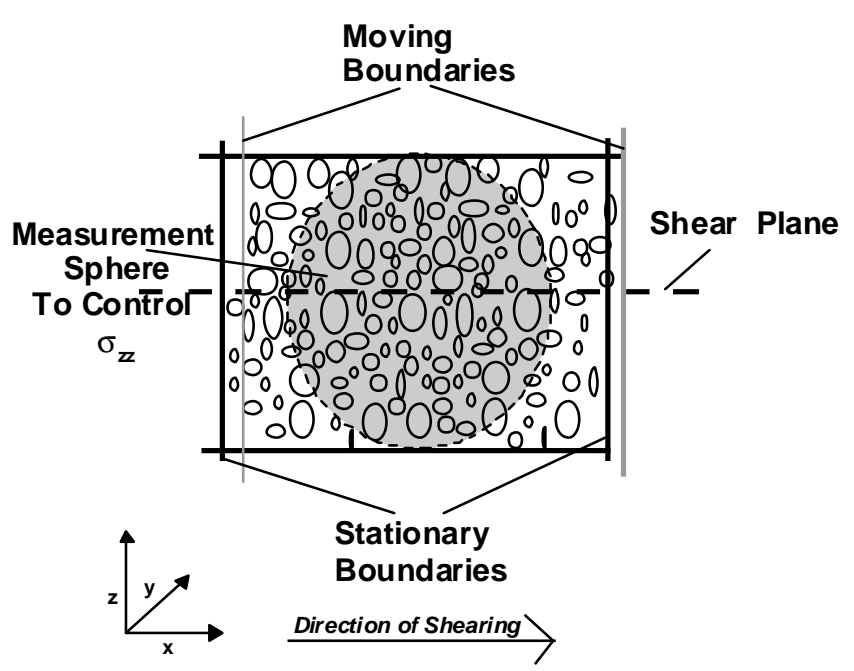

Figure 3: Illustration of implementation of direct shear tests in code 3DDEM.

\subsection{Analysis Parameters}

Table 3 lists the input parameters used in the simulation. A comparison of the parameters listed in Table 3 and Table 1 illustrates that the physical test and numerical simulation are equivalent, apart from the differences in density value used. Density scaling is commonly used to reduce the computational cost associated with DEM simulations for quasi-static analysed (e.g. Morgan and Boettcher (1999), Thornton (2000), O'Sullivan et al (2004)). The maximum stable time increment for explicit DEM simulations is proportional to the square root of the particle mass, motivating the use of such scaling. In the current analyses attempts were made to run the simulations without any density scaling. 
However, significant difficulties were encountered in bringing the system into equilibrium. These problems may have been a cons equence of the small particle masses used in comparison with the contact stiffnesses. Note that O'Sullivan (2002) performed a detailed analysis of the sensitivity of simulations of quasistatic triaxial tests to the input parameters. The results of this analysis indicated that once the ratio s $\sigma^{\text {confining }} / \mathrm{K}$ and $\rho / \mathrm{K}$ remained constant there was no variation in the simulation results $\left(\sigma^{\text {confining }}\right.$ $=$ confining pressure, $\mathrm{K}=$ linear contact spring stiffness, $\rho=$ particle density).

Previous analyses by O'Sullivan et al (2004) that considered specimens with re gular packing configurations confirmed that these precision steel ball particles can be modelled using uniform spheres. While some variation in the coefficient of friction values for the particles was measured, such variation has a negligible influence on the specimen response for specimens with irregular packing configurations (O'Sullivan et al 2002). For the analyses discussed in this paper the ball-boundary coefficient of friction was assumed to be zero. In discrete element simulations of laboratory tests we can easily examine the influence of boundary friction values on the macroscale response. Future analyses will explore this issue in more detail.

\begin{tabular}{|l|l|l|}
\hline Parameter & Value & Unit \\
\hline Radius & 0.9922 & $\mathrm{~mm}$ \\
\hline Density & $7.8334 \times 10^{3}$ & $\mathrm{~kg} / \mathrm{mm}^{3}$ \\
\hline Shear modulus & $7.945 \times 10^{7}$ & $\mathrm{~kg} /\left(\mathrm{mm} \mathrm{s}^{2}\right)$ \\
\hline Poisson ratio & 0.28 & \\
\hline $\begin{array}{l}\text { Friction coeffi- } \\
\text { cient }\end{array}$ & $5.5^{\circ}$ & \\
\hline
\end{tabular}

Table 3: Input parameters for DEM simulations

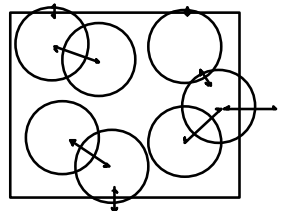

(a)

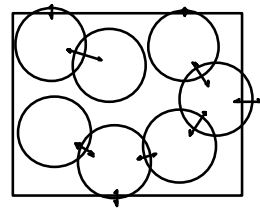

(b)

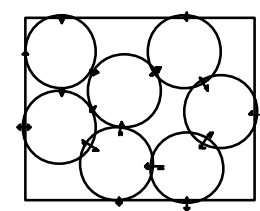

(c)
Figure 4: Illustration of specimen generation phase of analy sis

\subsection{Specimen generation}

The difficulties associated with generating a three-dimensional specimen to a specific void ratio for use in a discrete element analysis cannot be overstated. A review of some of the available approaches to specimen generation is provided by Cui and O'Sullivan (2003). As noted by O'Sullivan (2002), considerable effort has been expended by mathematicians to develop algorithms to generate dense, random assemblies of spheres (e.g. Zong (1999)). For the current analysis one such algorithm, proposed by Jodrey and Tory (1985), was initially used to generate the specimen. The resultant specimen was however less dense than the specimens tested in the lab (with a void ratio of 0.77 in comparison to void ratios of $0.58-0.61$ ). To overcome this limitation and achieve the required density value, 11700 balls with a radius of $0.9393 \mathrm{~mm}$ were initially generated in a box of size $0.06 \times 0.06$ $\mathrm{x} 0.02 \mathrm{~mm}$. This radius was then gradually expanded to $0.9922 \mathrm{~mm}$, the box height was increased to 21 $\mathrm{mm}$, and a DEM simulation was run until the system came into equilibrium. During this simulation the inter-particle friction angle was assigned a value of zero. The behavior of the particles during the equilibrium phase can be understood by reference to Figure 4. Initially upon initial radius expansion there was significant inter-particle overlap, with a maximum particle overlap of approximately 0.1059 $\mathrm{mm}$ (Figure 4(a)). As the equilibrium phase of the analysis progressed the balls moved to assume a dense packing configuration with a minimum amount of particle overlap (Figure 4(c)). Prior to shearing the maximum overlap was approximately $1.73 \times 10^{-3} \mathrm{~mm}$.

\subsection{Direct Shear Test Simulations}

Three DEM simulations were performed with initial isotropic stress conditions of $54.5 \mathrm{kPa}, 109 \mathrm{kPa}$ and $163.5 \mathrm{kPa}$. These initial stress conditions were attained using the servo-controlled approach detailed above. The upper section of the box was moved at a velocity of $0.001 \mathrm{~mm} / \mathrm{sec}$. It is important in these servo-controlled tests to verify firstly that the specified stress conditions are maintained for the duration of the simulation. A period of initial iteration was required to determine the appropriate control parameters to meet this requirement. Figure 5 illustrates the vertical stress in the specimen during the simulations as a function of horizontal strain. Both the vertical stress measured in the measurement sphere as well as the vertical stress calculated from the measured boundary forces are illustrated. The measured stresses varied slightly during the simulations, with a maximum deviation of $5 \%$ from the specified value measured in the measurement sphere.

The macro-scale test results are summarized in Table 4 and Figure 6. Figure 6(a) is a plot of shear stress versus horizontal strain, and Figure 6(b) is a plot of peak shear stress versus normal stress. The results yield a value for the angle of internal friction of $19.6^{\circ}$, which compares with a value of $22.7^{\circ}$ as the minimum value obtained in the laboratory tests. 
The void ratios of the specimens in the DEM simulations were close to the minimum void ratio values of the test specimens. Hence, the DEM simulations were found to underestimate the strength of the ball assembly measured in the laboratory.

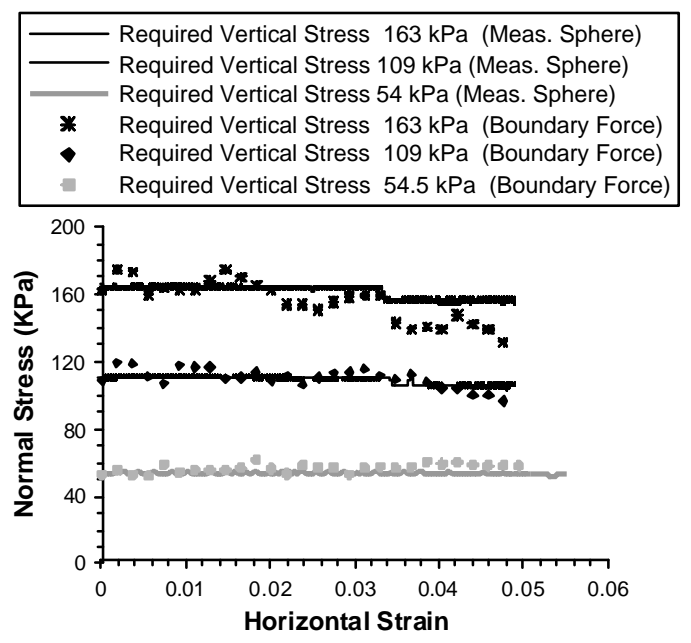

Figure 5: Normal Stresses $\left(\sigma_{\mathrm{zz}}\right)$ as calculated for measurement sphere and vertical stresses calculated fromboundary forces for the three simulations considered.

\begin{tabular}{|l|l|l|l|}
\hline Test No. & $\begin{array}{l}\text { Normal } \\
\text { Stress, } \mathrm{kPa}\end{array}$ & $\begin{array}{l}\text { Peak Shear } \\
\text { Stress, } \mathrm{kPa}\end{array}$ & Void Ratio \\
\hline 1 & 54.5 & 15.9 & 0.587 \\
\hline 2 & 109 & 37.5 & 0.587 \\
\hline 3 & 163.5 & 60.4 & 0.587 \\
\hline
\end{tabular}

Table 4: Summary of test simulation results.

A comparison of Figure 2 and Figure 6 indicates that the numerical simulations exhibit a significantly stiffer response in comparison to the physical tests. Considering the volumetric strain esponse of the physical test specimens, some compression was observed at the start of shearing prior to dilation. However for the numerical simulations the specimens dilated from the onset of shearing. These differences in volumetric strain response, suggest that there may be significant differences between the fabric of the physical test pecimens and the fabric of the specimens analyzed using DEM. In physical tests, O'Sullivan (2002) observed that the response of specimens of uniform steel balls is very sensitive to the specimen preparation approach; specimens with similar void ratios can exhibit different responses depending upon the specimen preparation approach used. Future research will address the source of these discrepancies considering the sensitivity of the response to the specimen preparation method as well as any compliance of the laboratory apparatus. As a quality assurance procedure the tests will be replicated using an alternative direct shear box located in another laboratory. Further analyses will explore the influence of ball-boundary friction on the macro-scale response as well as the contact constitutive model used in the simulations.

Coupling physical tests and numerical simulations is non-trivial as it can be difficult to replicate the exact details of the physical test in the numerical simulation. O'Sullivan (2002) describes some of the challenges associated with successful validation of discrete element codes using physical tests.
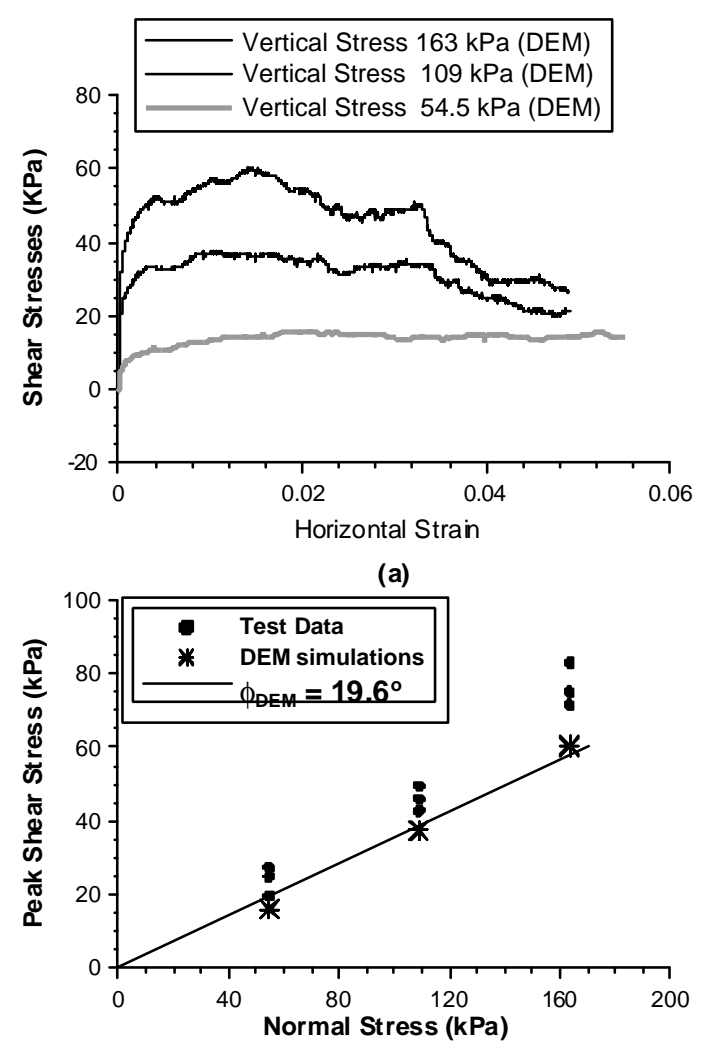

(b)

Figure 6: Su mmary of DEM simulation results. (a) Shear stress versus horizontal strain. (b) Peak shear stress versus normal stress

Notwithstanding the discrepancies between the stiffness observed in the laboratory tests and the numerical simulations, the numerical simulations clearly captured some of the typical response characteristics of granular materials in direct shear tests. For example, the stress-dependency of the specimen response can clearly be seen with the ratio of the peak shear stress to the residual shear stress increasing as the normal stress ncreases. Such stress dependency, however, was not observed in the physical tests. It is also interesting to observe in Figure 6(a) that the three specimens tested in the direct shear test appear to approach a similar value of shear stress (about 20kPa) as shearing progresses to large displacements. 


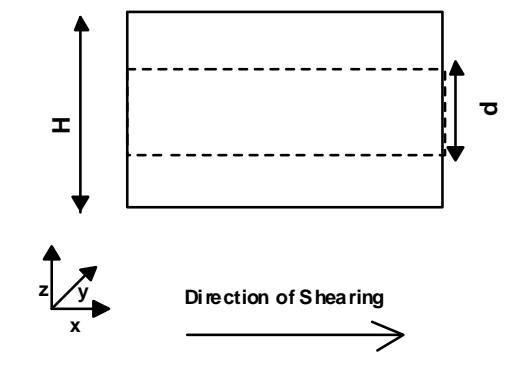

Figure 7: Illustration of configuration of rectangular box used to calculate local stresses

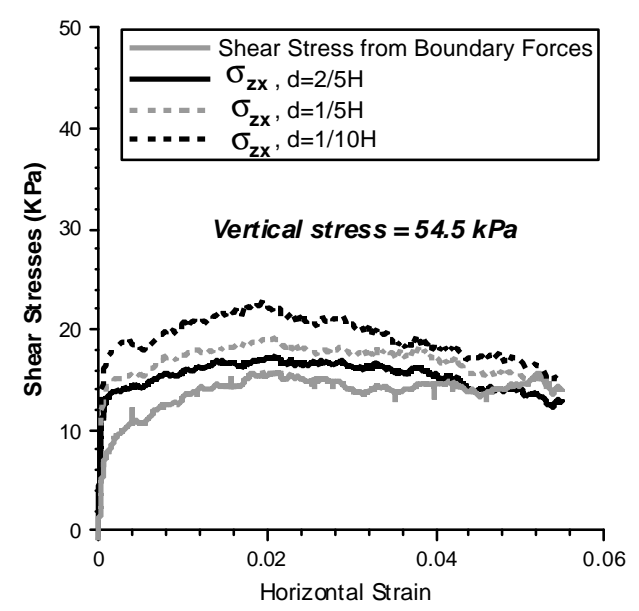

(a)

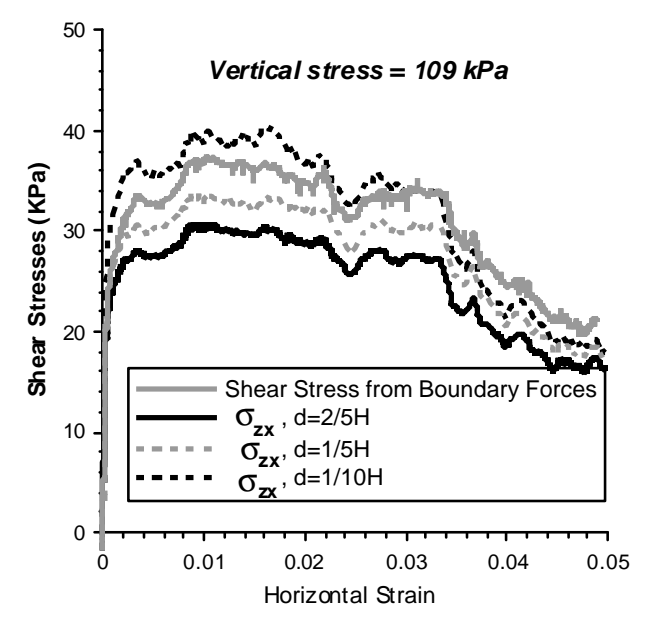

(b)

Figure 8: Variation of shear stress $\left(\sigma_{\mathrm{zx}}\right)$ values, measured in measurement boxes, as a function of horizontal strain. (a) Vertical stress $=54.5 \mathrm{kPa}$. (b) Vertical stress $=109 \mathrm{kPa}$.

\section{ANALYSIS OF MICRO-SCALE PARAMETERS}

\subsection{Stress analysis}

In a direct shear test the shear stress along the region of localization is inferred from the macro-scale measurement of shear force. In the DEM analyses a series of rectangular boxes were created and the average stresses within this box were calculated using Equation 1. The configuration of these boxes is illustrated in Figure 7; the center of the stress measurement box was located at the center of the specimen and the thickness of the box, $\mathrm{d}$, ranged from $\mathrm{H} / 10$ to $2 \mathrm{H} / 5$, where $\mathrm{H}$ is the specimen height. The variation of the local stresses, $\sigma_{\mathrm{xz}}$, as measured in the stress measurement box, with increasing strain is illustrated in Figure 8.

As illustrated in both Figure 8(a) and Figure 8(b), the shear stress, $\sigma_{\mathrm{zx}}$, measured increased as the thickness of the measurement box decreased. For an applied vertical stress of $54 \mathrm{kPa}$ (Figure 8(a)), the $\sigma_{\mathrm{zx}}$ values consistently exceeded the global shear stress values (calculated from the boundary forces). However, for an applied vertical stress of $109 \mathrm{kPa}$ (Figure 8(b)) and an applied vertical stress of 163 $\mathrm{kPa}$ (not shown), the bcal shear stress $\left(\sigma_{\mathrm{zx}}\right)$ values were less than the global shear stress. For the three vertical stresses considered, the ratio of the peak $\sigma_{\mathrm{zx}}$ value for $\mathrm{d}=1 / 5 \mathrm{H}$ divided by the peak $\sigma_{\mathrm{zx}}$ value for $\mathrm{d}=2 / 5 \mathrm{H}$ is consistently 1.1 , whereas the ratio of the peak $\sigma_{\mathrm{zx}}$ value for $\mathrm{d}=1 / 10 \mathrm{H}$ divided by the peak $\sigma_{\mathrm{zx}}$ value for $\mathrm{d}=2 / 5 \mathrm{H}$ is consistently 1.3 .

\subsection{Incremental Displacements}

A three-dimensional plot of the incremental displacements for the simulation with a normal stress of $54 \mathrm{kPa}$ is illustrated in Figure 9 for the horizontal strain increment 0.003 to 0.052 . Figure 9(a) is a front view of the specimen and Figure 9(b) is a side view of the specimen. For ease of visualization only displacements exceeding the average incremental displacement are illustrated. Referring firstly to Figure 9(a), as would be expected, most of the displacement is concentrated in the uper half of the shear box. There also is a concentration of diplacement vectors close to the left and right boundaries. At the left hand side of the box there is significant downward motion of the particles. The magnitude of the upward displacement of the particles in the upper right section of the box is less than the magnitude of the downward motion of the parti- 
cles close to the left boundary. Comparing Figure 9(a) and Figure 9(b), it is clear that the components of the particle displacement vectors in the $y$ direction are small in comparison to the component of the vectors in the $\mathrm{z}$-direction.

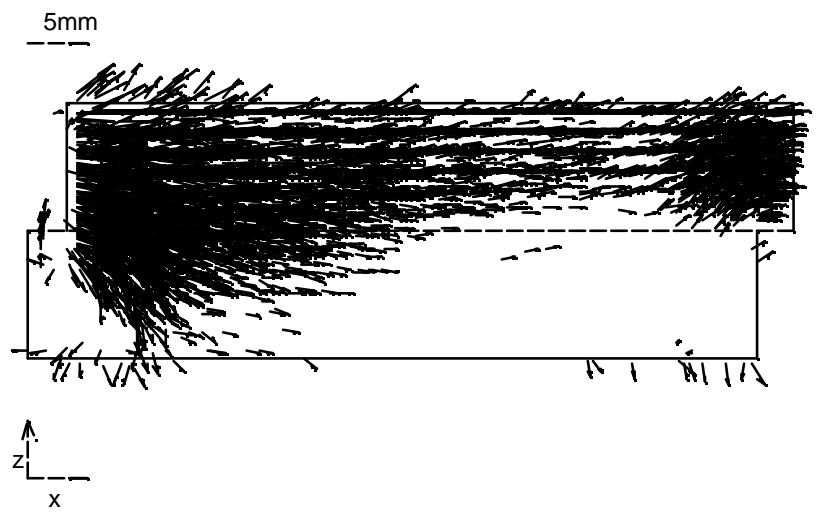

(a)
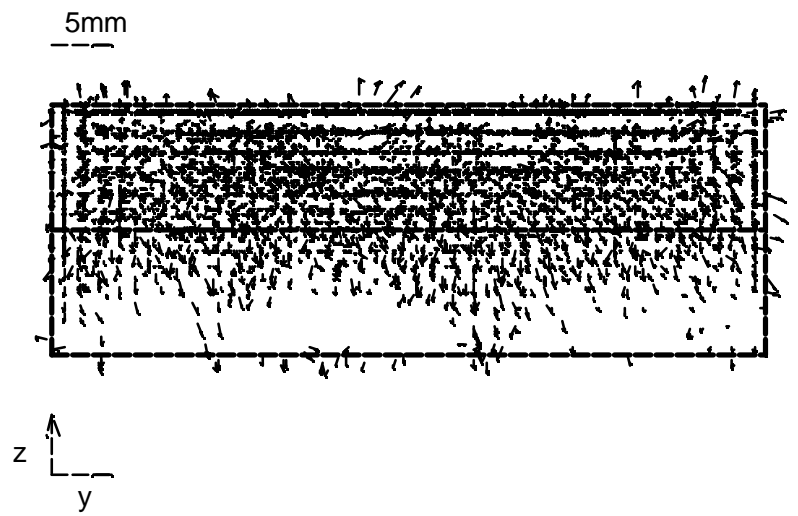

(b)

Figure 9: Incremental displacement vectors for horizontal strain increment of 0.003 to 0.052 , vertical stress $54.5 \mathrm{kPa}$. (a) Front view (looking along y axis) (b) Side view (looking along $\mathrm{x}$ axis). Note: For ease of visualization only displacements exceeding the mean displacement value are illustrated.

\subsection{Contactforces}

In order to appreciate the spatial variation in the contact forces, a three-dimensional plot of the unit contact force vectors for the simulation with a normal stress of $54 \mathrm{kPa}$ at a horizontal strain of 0.052 is illustrated in Figure 10. Figure 10(a) is a front view of the specimen and Figure 10(b) is a side view of the specimen. For ease of visualisation, only contacts where the magnitude of the contact force exceeds the average contact force +1 standard deviation are considered. The distribution of contact forces illustrated in Figure 10(a) is qualitatively similar to the distribution of contact forces obtained by Zhang and Thornton (2002) in their twodimensional discrete element analysis of the direct shear test. The forces are transmitted diagonally across the specimen. An orthogonal view of the specimen, along the $\mathrm{z}$ axis, is given in Figure 10(b), however, it is difficult to identify any clear trend in the orientation of the contact force vectors from this perspective.

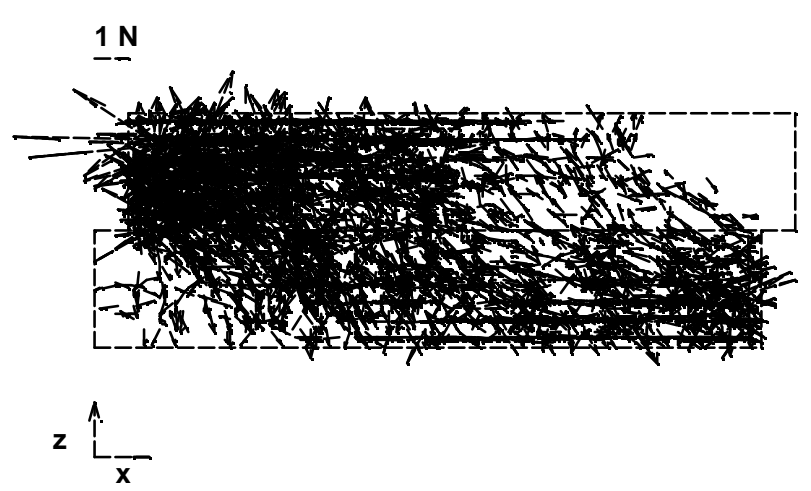

(a)

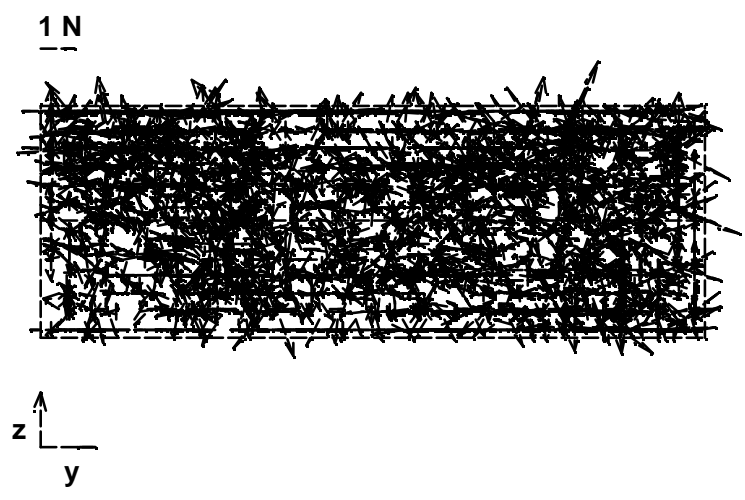

(b)

Figure 10: Contact force vectors at a horizontal strain of 0.052 , vertical stress $54.5 \mathrm{kPa}$. (a) Front view (looking along y axis) (b) Side view (looking along x axis). Note: For ease of visualization only contacts where the forces exceeds the average contact force +1 standard deviation are considered.

Figure 11 is a three dimensional plot of the contact force vectors, giving an indication of both the magnitude and direction of the contact forces. As illustrated in Figure 11(a) it is clear that the $x$ component of the contact force exceeds the $z$ component. Considering the distribution of the $y$ and $\mathrm{z}$ components of the contact forces, the plot is approximately circular, as illustrated in Figure 11(b). The $\mathrm{y}$ and $\mathrm{z}$ components of the contact forces are therefore relatively evenly distributed. An analysis of the local normal stresses as calculated from the contact forces indicated that $\sigma_{\mathrm{xx}}>\sigma_{\mathrm{yy}}>\sigma_{\mathrm{zz}}$. The relatively large value in $\sigma_{\mathrm{yy}}$ is an indicator of the three dimensional nature of the problem. 


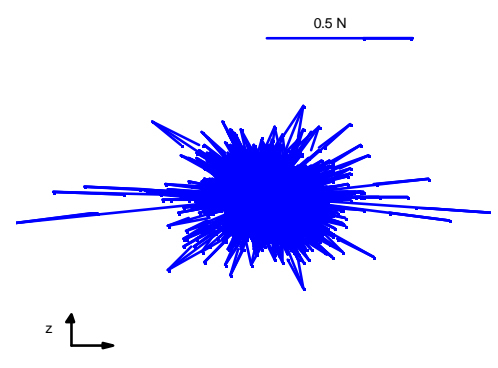

(a)

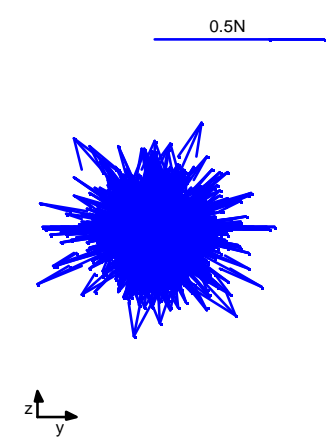

(b)

Figure 11: Three dimensional plot of contact force vectors (a) Front view (looking along y axis) (b) Side view (looking along $\mathrm{x}$ axis).

\subsection{Strain localization analysis}

For the simulation with a vertical stress of $163 \mathrm{kPa}$, the local strain values were calculated using the nonlinear homogenization technique proposed by O'Sullivan et al (2003). The strain values are plotted on a vertical plane through the center of the specimen, i.e. with $y=30 \mathrm{~mm}$. Figure 12 illustrates the local strain values for the horizontal strain increment from 0.003 to 0.017 . While there are zones with high strain values at mid height close to the left and right boundaries, no clear localization through the center of the specimen is apparent, even though the specimen is close to the peak stress at this strain level. Considering the volumetric strain values $\left(\varepsilon_{\mathrm{vol}}\right)$, the localizations appear to be inclined. Similar inclined localizations were observed by Potts et al (1987) where a constitutive model without strain softening was used.

Figure 13 illustrates the distribution in strain values at a horizontal strain of 0.047 . The distribution of shear strains is highly iregular and there is still no clear evidence of a localization in the specimen. Similar strain distributions were observed for the specimens with vertical stresses of $54.5 \mathrm{kPa}$ and 109 $\mathrm{kPa}$. This contrasts with the study of localizations in specimens with regular packing configuration de- scribed by O'Sullivan and Bray (2003) where a distinct localization was observed in three-dimensional simulations of plane strain tests.
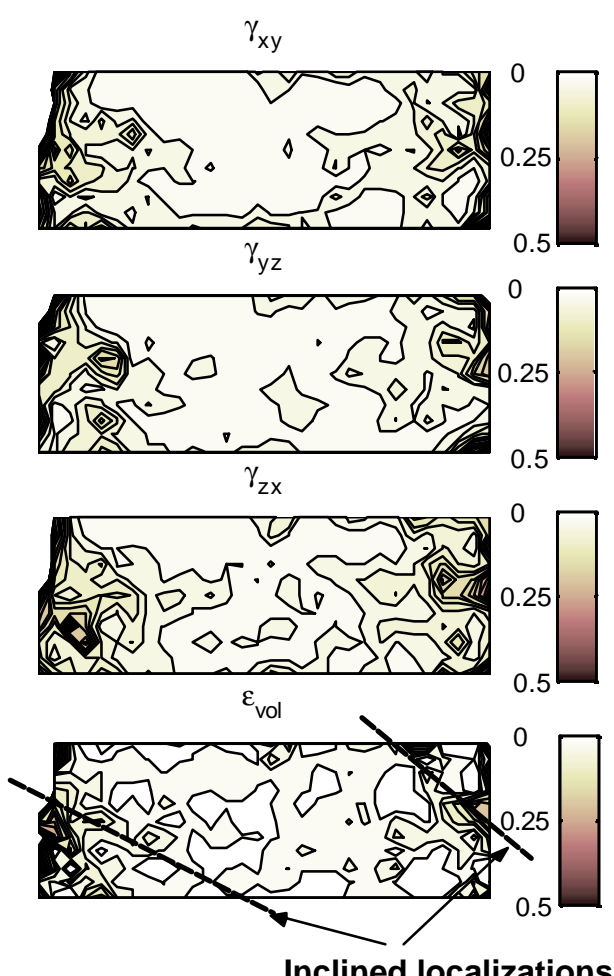

Inclined localizations

Figure 12: Strain contours for horizontal strain increment 0.003 to 0.017 , plotted on a vertical plane through center of specimen with $\mathrm{y}=30$. Normal stress $163 \mathrm{kPa}$. Contour interval 0.025 .

\section{DISCUSSION}

The direct shear test has been studied using discrete element analysis in combination with a series of complementary physical tests. The agreement between the laboratory tests and the numerical simulations was not wholly satisfactory. The angle of friction calculated using the results of the simulations was $3^{\circ}$ lower than that obtained in the physical tests. The response in the simulations was also stiffer than the response dbserved in the physical tests. Further tests and simulations are required to resolve these discrepancies, although it is likely that test device friction may be a contributing factor.

An analysis of the results of the simulations considered the local stress values, the incremental displacements, the distribution of contact forces and the local strain values. Whereas the particle displacements were predominantly restricted to the direction of shearing (i.e. parallel to the $\mathrm{y}=0$ plane), significant contact forces developed in the y-direction, itlustrating the three dimensional nature of the material response at the particle scale. Within the 
specimen, the stresses are non- uniform with the shear stresses increasing closer to the zone of shearing. The local strain values are non- uniform and the localization cannot be identified at horizontal strains of approximately $5 \%$.

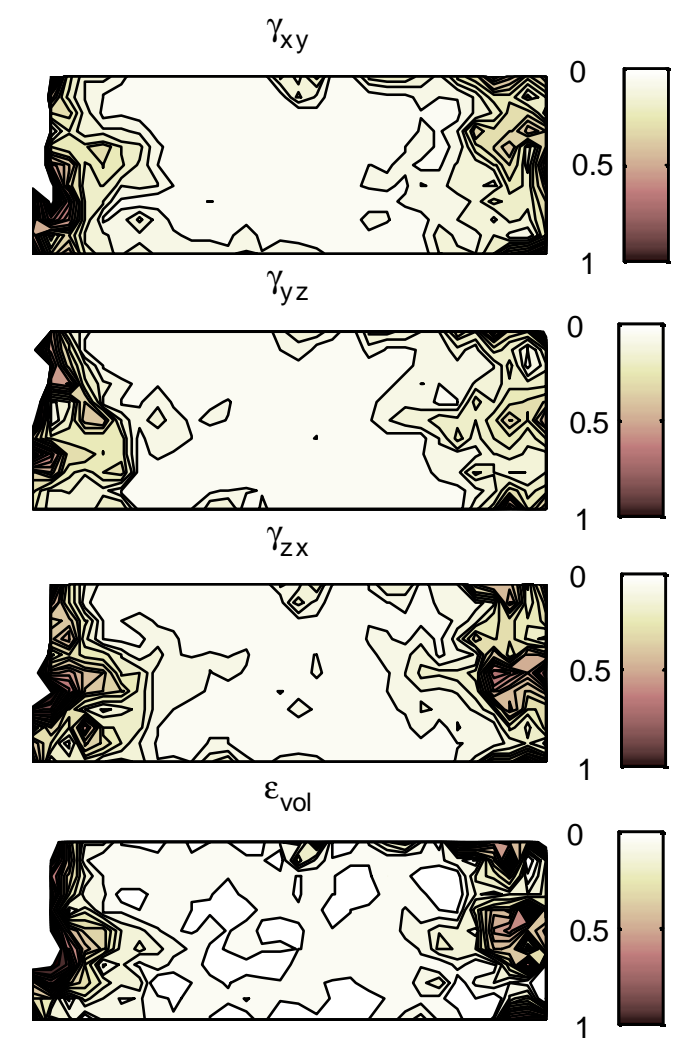

Figure 13: Strain contours for horizontal strain increment 0.003 to 0.047 , plotted on a vertical plane through center of specimen with $\mathrm{y}=30$. Normal stress $163 \mathrm{kPa}$. Contour interval 0.05 .

\section{ACKNOWLEDGEMENTS}

Funding for this research was provided by the Irish Research Council for Science, Engineering and Technology (IRCSET) under the Basic Research Grant Scheme. Additional partial funding was provided by the Institution of Engineers of Ireland Geotechnical Trust Fund. The authors are grateful to Mr. George Cosgrave, University College Dublin, for his assistance in performing the laboratory tests.

\section{REFERENCES}

ASTM (1990) D3080: standard test method for direct shear test of soils under consolidated drained conditions, Annual book of ASTM standards: 417-422

Cui, L. and C. O'Sullivan (2003) "Analysis of a Triangulation Based Approach for Specimen Generation for Discrete Element Simulations." Granular Matter V.5(3): 135145

Dounias, G.T. and Potts, D.M. (1993) “Numerical analysis of drained direct and simple shear test" Journal of Geotechnical Engineering Vol. 119, No. 12, 1870-1891
Holtz R. D. and Kovacs, W.D. (1981) An Introduction to Geotechnical Engineering Prentice Hall.

Itasca (2002). PFC2D Manual, Theory and Backround.

Iwashita, K. and Oda, M., " Micro-deformation mechanism of shear banding process based on modified distinct element method" ,Powder Technology 109: 192-205 (2000).

Jodrey, W.S. and Tory, E.M. (1985) " Computer simulation of close random packing of equal spheres" Physical Review A 32 (4): 2347-2351

Lings, M.L. and Dietz, M.S. (2004) "An improved direct shear apparatus for sand" Geotechnqiue Vol. 54 No. 4 245-256

Masson, S. and Martinez, J. (2001) "Micromechanical analysis of the shear behavior of a granular material" ASCE Journal of Engineering Mechanics, Vol. 127, No. 10: 1007-1016

Morgan, J. and Boettcher, M.S. (1999) “ Numerical simulations of granular shear zones using the distinct element method. 1. Shear zone kinematics and the micromechanics of loca 1ization" Journal of Geophysical Research, Vol. 104, No. B2: 2703-2719

Morgan, J. (1999) “ Numerical simulations of granular shear zones using the distinct element method. 2. Effects of part icle size distribution and interparticle friction on mechanical behavior" Journal of Geophysical Research, Vol. 104, No. B2: 2721-2732

O'Sullivan, C. (2002). The Application of Discrete Element Modelling to Finite Deformation Problems in Geomechanics. Ph.D. thesis, Dept. of Civ. Engrg., Univ. of California, Berkeley.

O'Sullivan, C., J.D. Bray, and M.F. Riemer, (2002) The Influence of Particle Shape and Surface Friction Variability on Macroscopic Frictional Strength of Rod-Shaped Particulate Media" ASCE Journal of Engineering Mechanics, Vol. 128, No. 11. p 1182-1192

O'Sullivan, C. and J.D. Bray (2003) “ Evolution of Localizations in Idealized Granular Materials" Workshop on Quasistatic Deformations of Particulate Materials

O'Sullivan, C., Bray, J.D. and M.F. Riemer, (2004) "An examination of the response of regularly packed specimens of spherical particles using physical tests and discrete element simulations." ASCE Journal of Engineering Mechanics, Vol. 130, No. 10, in press.

O'Sullivan, C., Bray, J. D. and Li, S. (2003) “A New Approach for Calculating Strain for Particulate Media" International Journal for Numerical and Analytical Methods in Geomechanics Vol. 27 No. 10.: 859-877.

Potts, D. M., Dounias, G.T., and Vaughan, P. R. (1987) “ Finite element analysis of the direct shear box test" Geotechnique, Vol. 37, No. 1: 11-23

Skempton, A. W. (1949) Alexandre Collin: a note on his pioneer work in soil mechanics, Geotechnique, Vol. 19., No. 1: $75-86$

Thomas, P.A. (1997) Discontinuous deformation analysis of particulate media. Ph.D. thesis, Dept. of Civ. Engrg., Univ. of California, Berkeley.

Toolan, F.E. (1987) Preface to "Syposium in print on the engineering application of direct and simple shear testing", Geotechnique 1987, Vol. 37, No. 1, 1-2.

Shibuya, S., Mitachi, T., and Tamate, S. (1997) "Interpretaion of direct shear box testing of sands as quasi-simple shear" Geotechnique 47(4): 769-790

Zhang, L and Thornton, C. (2002a). "DEM simulations of the direct shear test". Proceedings of $15^{\text {th }}$ ASCE Engineering Mechanics Conference, June2-5, 2002. Columbia University, New York, NY.

Zong, C. (1999) Sphere Packings, Springer. 